\title{
NỒNG ĐỘ THIAMIN HUYẾT TƯƠNG Ở BỆNH NHÂN ĐÁI THÁO ĐƯờNG TÍP 2
}

Nguyễn Thu Phuơng ${ }^{1}$, Hoàng Trung Vinh ${ }^{2}$

DOI: $10.47122 / v j d e .2020 .45 .1$

1. Bệnh viện 199 - Bộ Công an; 2. Học viện Quân y

\section{TÓM TẮT}

Mục tiêu: Khảo sát nồng độ thiamin huyết tương và tỷ lệ biến đổi của chỉ số ở bệnh nhân đái tháo đường típ 2 (BN ĐTĐT2). Đối tượng và phưong pháp: 254 đối tượng chia thành 2 nhóm bao gồm 161 BN ĐTĐT2 và 93 đối tượng thuộc nhóm chứng được xét nghiệm nồng độ thiamin huyết tương bằng phương pháp ELISA. Kết quả: Nồng độ thiamin ở BN thấp ở có ý nghĩa so với nhóm chứng. Tỷ lệ BN giảm thiamin là $86,3 \%$. Nồng độ thiamin và tỷ lệ giảm thiamin ở $\mathrm{BN}$ nam và nữ, giữa các nhóm tuổi tương đương nhau. Kết luận: $\mathrm{BN}$ ĐTĐT2 biểu hiện giảm nồng độ thiamin. Tỷ lệ đối tượng giảm gặp ở mức cao. Không có sự khác biệt về nồng độ thiamin giữa nam và nữ cũng như giữa các nhóm tuổi.

Tù khóa: Đái tháo đường típ 2, nồng độ thiamin.

\section{ABSTRACT \\ Plasma thiamin level in type 2 diabetes mellitus patients \\ Nguyen Thu Phuong ${ }^{1}$, Hoang Trung Vinh ${ }^{2}$ 1. 199 Hospital - Ministry of Public Security; \\ 2. Vietnam Military Medical University}

Objectives: To investigate plasma thiamine level and rates of its changes in patients with type 2 diabetes mellitus (T2DM patients). Subjects and methods: 254 participants divided into 2 groups including 161 T2DM patients in the study group and 93 individuals in the control group were measured plasma thiamin level by ELISA method. Results: Thiamine level in T2DM patients was significantly lower than that in the control group. The rate of patients with thiamin reduction was $86.3 \%$. Thiamin level and decreased thiamin rate were similar between male and female patients, between age groups. Conclusions: Plasma thiamin level was decreased in T2DM patients. Patients with reduced thiamin level was seen with a high rate. There were no significant differences in thiamine levels between men and women as well as between age groups.

Keywords: Type 2 diabetes mellitus, thiamin level.

Chịu trách nhiệm chính: Hoàng Trung Vinh

Ngày nhận bài: 5/1/2021

Ngày phản biện khoa học: 11/1/2021

Ngày duyệt bài: 4/3/2021

Email: hoangvinh.hvqy@gmail.com

ĐT: 0903201250

\section{1. ĐẶT VẤN ĐỀ}

Thiamin hay còn gọi là vitamin $\mathrm{B} 1$ là chất xúc tác quan trọng trong nhiều phản ứng sinh học của cơ thể. Thiamin không được tự tổng hợp mà phải đưa từ ngoài vào qua đường ăn uống. Bệnh nhân ĐTĐT2 do nhiều nguyên nhân khác nhau thường có biểu hiện thiếu hụt các nguyên tố vi lượng trong đó có thiamin. Thiếu thiamin ảnh hưởng đến nhiều biểu hiện của $\mathrm{BN}$ trong đó rõ nét nhất là tổn thương thận, bệnh thần kinh ngoại vi, biến chứng tim. Chính vì vậy trong điều trị cần được bồi phụ thiamin đầy đủ. Để biết được nồng độ thiamin ở BN ĐTĐT2, đề tài đã được thực hiện nhằm mục tiêu: Khảo sát nồng độ thiamin huyết tuoong và tỷ lệ biến đổi của chì số ở bệnh nhân đái tháo đường típ 2.

\section{2. ĐỐI TƯợNG VÀ PHƯƠNG PHÁP NGHIÊN CÚU}

+ 161 BN ĐTĐT2 được chẩn đoán lần đầu hoặc đã được chẩn đoán, điều trị trước thời điểm nghiên cứu.

+ 93 đối tượng thuộc nhóm chứng khỏe mạnh có tuổi, giới tương đồng với nhóm bệnh.

+ Tất cả các đối tượng được xét nghiệm nồng độ thiamin huyết tương bằng phương pháp ELISA theo quy trình chuẩn. Đơn vị tính: pg/ml.

+ Số liệu thu được xử lý bằng thuật toán thống kê với các phương pháp phù hợp. 


\section{KẾT QUẢ NGHIÊN CÚU}

Bảng 3.1. So sánh nồng độ thiamin giữa hai nhóm

\begin{tabular}{|l|c|c|c|}
\hline \multicolumn{1}{|c|}{ Thiamin (pg/ml) } & Nhóm chứng (n=93) & Bệnh nhân (n=161) & p \\
\hline Trung bình & $61,19 \pm 25,71$ & $41,39 \pm 28,31$ & \\
\hline Trung vị & 53,18 & 38,19 & $<0,001$ \\
\hline Min & 38,64 & 10,12 & \\
\hline Max & 221,95 & 269,24 & \\
\hline Tỷ lệ giảm (\%) & $\begin{array}{c}\text { Cut off tương ứng 5\% giá trị } \\
\text { thuộc nhóm chứng < 43,13 } \\
\text { pg/ml }\end{array}$ & $\mathbf{8 6 , 3}$ & \\
\hline
\end{tabular}

Nhận xét: Nồng độ thiamin huyết tương ở bệnh nhân đái tháo đường típ 2 thấp hơn có ý nghĩa so với nhóm chứng. Nồng độ thiamin của các đối tượng thuộc cả hai nhóm dao động trong một miền rất rộng.

Bảng 3.2. Nồng độ thiamin ở $B N$ nam và nữ $(n=161)$

\begin{tabular}{|c|c|c|}
\hline Giới & Trung vị $\mathbf{( p g / m l )}$ & Tỷ lệ giảm (n, \%) \\
\hline Nam $(\mathrm{n}=89)$ & $38,2(31,1-43,4)$ & $77(86,5)$ \\
\hline Nữ $(\mathrm{n}=72)$ & $38,2(33,3-42,9)$ & $62(86,1)$ \\
\hline $\mathbf{p}$ & $>0,05$ & $>0,05$ \\
\hline
\end{tabular}

Nhận xét: Nồng độ thiamin huyết tương, tỷ lệ giảm thiamin ở đối tượng nam và nữ khác biệt không có ý nghĩa thống kê.

Bảng 3.3. Nồng độ thiamin theo nhóm tuổi $(n=161)$

\begin{tabular}{|c|c|c|}
\hline \multicolumn{1}{|c|}{ Nhóm tuổi (năm) } & Trung vị (pg/ml) & Tỷ lệ giảm (n, \%) \\
\hline $40-49(\mathrm{n}=13)$ & $35,3(31,0-39,6)$ & $13(100,0)$ \\
\hline $50-59(\mathrm{n}=38)$ & $38,1(31,2-42,9)$ & $35(92,1)$ \\
\hline $60-69(\mathrm{n}=71)$ & $38,2(28,9-43,1)$ & $59(83,1)$ \\
\hline$\geq 70(\mathrm{n}=39) \quad \mathrm{p} \quad$ & $39,9(35,3-43,4)$ & $32(82,1)$ \\
\hline \multicolumn{1}{|c|}{$\mathrm{p}$} & $>0,05$ & $>0,05$ \\
\hline
\end{tabular}

Nhận xét: Nồng độ thiamin huyết tương, tỷ lệ giảm thiamin giữa các nhóm tuổi khác biệt không có ý nghĩa thống kê.

\section{BÀN LUẦN}

Nồng độ thiamin huyết tương của toàn bộ $\mathrm{BN}$ nghiên cứu cũng tương tự như nhóm chứng về khía cạnh phân bố. Với $161 \mathrm{BN}$ có giá trị thiamin huyết tương phân bố trong miền rất rộng, theo đó giá trị nhỏ nhất là $10,12 \mathrm{pg} / \mathrm{ml}$, lớn nhất là 269,24 pg/ml. Chính vì vậy mặc dù cũng xác định được giá trị trung bình của nồng độ song do các chỉ số phân bố không tuân theo luật chuẩn nên không thể so sánh được dựa vào giá trị trung bình mà phải xác định trung vị tương tự như ở nhóm chứng.

Qua tính toán nhận thấy nồng độ thiamin huyết tương của BN ĐTĐT2 là $38,19 \mathrm{pg} / \mathrm{ml}$, thâp hơn có ý nghĩa so với giá trị tương ứng của nhóm chứng với $\mathrm{p}<0,001$. Qua đó nhận thấy nồng độ thiamin huyết tương ở $\mathrm{BN}$ ĐTĐT2 thuộc nhóm nghiên cứu giảm so với nhóm chứng. Giải thích về nguyên nhân giảm nồng độ thiamin huyết tương $\mathrm{BN}$ ĐTĐ nói chung và ĐTĐT2 nói riêng đã được nhiều tác giả đề cập. Mặc dù có giả thuyết về cơ chế gây giảm thiamin chưa được thống nhất nhưng có một đặc điểm chung là trong nghiên cứu của bất kỳ tác giả nào ở $\mathrm{BN} Đ \mathrm{D} Đ$ đều nhận thấy giảm nồng độ thiamin so với nhóm chứng.

Đa số các tác giả đều nhận thấy ở $\mathrm{BN}$ ĐTĐT2 đều có giảm nồng độ thiamin huyết tương do nhiều nguyên nhân khác nhau. Tuy vậy số liệu của các tác giả đưa ra cũng có sự khác nhau tùy thuộc vào đối tượng quan sát. 
Bảng 4.1. Nồng độ thiamin huyết tương ở BN ĐTĐT2 của một số tác giả

\begin{tabular}{|l|c|c|c|}
\hline \multicolumn{1}{|c|}{ Tác giả } & BN ĐTĐT2 & Nhóm chứng & p \\
\hline Alam S, et al (2012) [1] & $\begin{array}{c}10,8(0,8-84,5) \\
7,1(1,1-31,3)\end{array}$ & $\begin{array}{c}64,1 \mathrm{nmol} / 1 \\
44(63-93,7)\end{array}$ & \\
\hline Hobara R, et al (1981) [2] & $28 \mathrm{pg} / \mathrm{ml}$ & & \\
\hline Daghri N, et al (2015) [3] & $33,6 \pm 16,5$ & $42,9 \pm 15,1 \mathrm{ng} / \mathrm{ml}$ & 0,002 \\
\hline Alkhalaf A, et al (2010) [4] & $31,8 \pm 7,7 \mathrm{nmol} / 1$ & $64,1 \pm 12,0 \mathrm{nmol} / 1$ & \\
\hline Yuka K, et al (2008) [5] & $94,1 \pm 3,1 \mathrm{nmol} / 1$ & $286,6 \pm 60,1 \mathrm{nmol} / 1$ & \\
\hline Anwar A, et al (2020) [6] & $14,89 \pm 4,82 \mathrm{pg} / \mathrm{ml}$ & $\begin{array}{c}69,56 \pm 25,71(\mathrm{trung} \\
\text { vị: } 53,18) \mathrm{pg} / \mathrm{ml}\end{array}$ & $<0,001$ \\
\hline Kết quả NC (2020) & $38,19 \mathrm{pg} / \mathrm{ml}$ & $53,18 \mathrm{pg} / \mathrm{ml}$ & $<0,001$ \\
\hline
\end{tabular}

Cùng với giảm nồng độ thiamin huyết tương, ở BN ĐTĐT2 còn cho thấy tỷ lệ giảm giá trị tuyệt đối nồng độ của các đối tượng cũng nhận thấy ở mức cao. Nếu xác định cut off là vị trí tương ứng $5 \%$ giá trị thuộc nhóm chứng $<43,13 \mathrm{pg} / \mathrm{ml}$ thì sẽ có $86,3 \%$ trường hợp giảm nồng độ. Song nếu cut off là tứ phân vị dưới $(25 \%)$ số đối tượng của nhóm chứng $<48,7 \mathrm{pg} / \mathrm{ml}$ sẽ có $95,7 \%$ trường hợp giảm thiamin. Số liệu quan sát của các tác giả đưa ra tỷ lệ giảm thiamin ở $\mathrm{BN}$ ĐTĐT2 rất khác nhau, tùy thuộc vào đối tượng nghiên cứu được lựa chọn. Tuy vậy có thể nói đa số tác giả đều nhận thấy BN ĐTĐT2 có tỷ lệ cao biểu hiện giảm nồng độ thiamin huyết tương.

Bảng 4.2. Tỷ lệ BN ĐTĐT2 biểu hiện giảm thiamin huyết tương của một số tác giả

\begin{tabular}{|l|l|}
\hline \multicolumn{1}{|c|}{ Tác giả } & \multicolumn{1}{c|}{ Tỷ lệ giảm thiamin } \\
\hline Nix A, et al (2014) [7] & MAU (-): 98\% \\
MAU (+): $100 \%$
\end{tabular}

Qua bảng kết quả trên nhận thấy những quan sát của một số tác giả đưa ra tỷ lệ giảm nồng độ thiamin ở BN ĐTĐT2 đều ở mức cao nhưng rất dao động. Khi đã có tổn thương thận với MAU (+) thì tỷ lệ giảm thiamin có thể lên đến $100 \%$. Đây có lẽ cũng là bằng chứng liên quan đến giảm thiamin huyết tương là do tổn thương thận.

\section{KẾT LUẬN}

+ Nồng độ thiamin giảm có ý nghĩa so với nhóm chứng $(38,19 \mathrm{pg} / \mathrm{ml}$ so với 53,18 pg/ml) $(\mathrm{p}<0,001)$.

+ Tỷ lệ bệnh nhân giảm thiamin nói chung: $86,3 \%$.

+ Nồng độ thiamin huyết tương ở bệnh nhân nam và nữ, giữa các nhóm tuổi khác biệt chưa có ý nghĩa thống kê ( $\mathrm{p}>0,05)$.

+ Tỷ lệ giảm thiamin ở bệnh nhân nam và nữ tương đương nhau $(86,5 \%$ và $86,1 \%$, $\mathrm{p}>0,05)$.

+ Tỷ lệ bệnh nhân giảm thiamin giữa các nhóm tuổi: 40-49; 50-59; 60-69; $\geq 70$ khác biệt không có ý nghĩa thống kê, tương ứng 100,0\%; $92,1 \% ; 83,1 \% ; 82,1 \%(\mathrm{p}>0,05)$.

\section{TÀI LIỆU THAM KHẢO}

1. Alam S, Riaz S, Akhtar M, et al. (2012) "Effect of High Dose Thiamine Therapy on Risk Factors in Type 2 Diabetes". Journal of Diabetes \& Metabolism, pp. $1 / 15-15 / 15$.

2. Hobara R, Ozawa K, Okazaki M, et al. (1981) "Ralationship between thiamine and glucose levels in diabetes mellitus". Japan J Pharmacol, pp.1098-1100. 
3. Daghri N, Alharbi M, Wani K, et al. (2015) "Biochemical changes correlated with blood thiamine and its phosphate esters levels in patiens with diabetes type 1 (DMT1)”. Int J Clin Exp Pathol, 8(10), pp.13483-13488.

4. Alkhalaf A, Klooster A, Oeveren W, et al. (2010) "A Double - Blind, Randomized, Placebo - controlled Clinical Trial on Benfotiamine Treatment in Patients With Diabetic nephropathy". Diabetes Care, pp.1598-1601.

5. Yuka K, Shirakawa H, Yamane K, et al. (2008). "Prevention of incipient diabetic cardiomyopathy by high-dose thiamine". The Journal of Toxicological Sciences (J.Toxicol. Sci), 33(4), pp.459-472.

6. Anwar A, Azmi MA, Siddiqui JA, et al (2020). "Thiamine level in type I and type II diabetes mellitus patients: $A$ Comparative study focusing on hematological and biochemical evaluations". Cureus, 12(5), pp.1/1111/11.

7. Nix A, Zirwes R, Bangert V, et al. (2014). "Vitamin B status in patients with type 2 diabetes mellitus with and without incipient nephropathy". Diabetes Research and Clinical Practice, pp.157165.

8. Marrs C (2015). "Diabetes and Thiamine: A Novel treatment opportunity”. Hormones Matter, pp.1/10$10 / 10$.

9. Esther G, Nanne K (2014). "Thiamine", Diapedia the living textbook of diabetes, no 8 , pp.1/4-4/4.

10. Richard H.Mann, (2018) "Thiamine deficiency and diabetic polyneuropathy". Compelling evidence for an interrelationship, 10(11), pp.1-19. 